\title{
Calorimetria indireta em pacientes oncológicos pediátricos
}

\author{
Indirect calorimetry in pediatric cancer patients
}

\section{DOI: $10.37111 /$ braspenj.2019344002}

Jaqueline Borges

Sabrina Galdino da Silva ${ }^{2}$

Thamyres Figueiredo ${ }^{3}$

Fernanda Jabur ${ }^{4}$

Daiane Ferreira da Silva

Andreia Ribeiro Pereira Aguiar de Paula ${ }^{6}$

Mariana dos Santos Murra ${ }^{7}$

\section{Unitermos:}

Calorimetria indireta. Oncologia. Pediatria.

\section{Keywords:}

Calorimetry, Indirect. Medical Oncology. Pediatrics.

\section{Endereço para correspondência:}

Jaqueline Borges

Av. Gabriel Garcia Leal, 1484 - Paranoá - Guaíra, SP,

Brasil - CEP:14790-000

E-mail: jack borges@hotmail.com

\section{Submissão}

6 de julho de 2019

\section{Aceito para publicação}

19 de setembro de 2019

\section{RESUMO}

Introdução: Pacientes oncológicos pediátricos são particularmente vulneráveis à desnutrição e apresentam suas necessidades elevadas devido à doença e ao seu tratamento. A determinação do gasto energético individualizado fornece necessidades precisas durante o tratamento, minimizando o risco nutricional. A calorimetria indireta $(\mathrm{Cl})$ ou equações preditivas têm sido sugeridas em pacientes críticos. Atualmente, o uso das fórmulas preditivas ainda é controverso, além disso, todas apresentam limitação e baixa acurácia quando comparadas à $\mathrm{Cl}$, apresentando, assim, grande probabilidade de hipo ou hiperalimentação. A Cl é considerada padrão-ouro para avaliar o gasto energético, mas nem sempre está disponível. As recentes diretrizes da Sociedade Americana de Nutrição Parenteral e Enteral para suporte nutricional da criança gravemente doente sugerem que medidas de $\mathrm{Cl}$ sejam obtidas quando possível em pacientes pediátricos com suspeita de alterações metabólicas ou desnutrição, segundo uma lista de critérios que podem levar à instabilidade metabólica, tornando as equações preditivas padronizadas ainda menos usadas. Embora o uso padrão da $\mathrm{Cl}$ seja limitado devido à disponibilidade do equipamento, à necessidade de equipe e ao custo, as medições tornaram-se mais precisas. O objetivo deste estudo foi comparar as medidas de gasto energético através da $\mathrm{Cl}$ com as equações preditivas. Método: Estudo prospectivo de caráter observacional, realizado em uma UTI oncológica pediátrica de janeiro a junho de 2019. Foram coletados dados como sexo, idade e antropometria de 18 pacientes que estavam dentro do critério de inclusão. As necessidades energéticas foram calculadas pelas equações preditivas utilizadas na rotina, como Holliday-Segar, ASPEN e Dietary Reference Intake (DRI), já a CI foi utilizada em pacientes em ventilação mecânica, estáveis hemodinamicamente, com $\mathrm{FiO}$ menor de $60 \%$ e a mesma foi acoplada no ventilador por 2 horas; após 2 horas foi feita a leitura do gasto energético no monitor. Os dados foram analisados descritivamente e pelo Índice de Concordância entre as fórmulas e a $\mathrm{Cl}$, sendo analisada através do teste de Spearman e a concordância por meio da correlação intraclasse, foi adotado significância 0,05. Resultados: Foram incluídos 18 pacientes, destes $77 \%$ eram do sexo feminino, a prevalência do estado nutricional foi a eutrofia $(44,4 \%)$, já os pacientes sobrepeso, baixo peso e desnutridos somaram $50,1 \%$. Foi analisada a correlação das fórmulas preditivas com a Cl, Holliday-Segar $(0,461-p=0,054)$, DRI $(0,428-p=0,076)$ e ASPEN $(0,232-p=0,354)$, sendo que a fórmula de Holliday-Segar foi a que mais concordou com o padrão-ouro. Conclusão: $\mathrm{A} \mathrm{Cl}$ deve ser utilizada, se disponível, quando o paciente estiver dentro dos critérios de inclusão, porém devido ao seu alto custo, as fórmulas preditivas podem ser utilizadas, considerando a importância das necessidades nutricionais.

\section{ABSTRAC}

Introduction: Pediatric cancer patients are particularly vulnerable to malnutrition and are subject to low demands due to the disease and its treatment. Determination of individualized energy expenditure is necessary to prevent damage during treatment while minimizing nutritional risk. Calorimetry indirect (IC) or predictive equations have been suggested in critically ill patients. Currently, the use of predictive formulas is still controversial, and all of them have limitation and low accuracy when compared to IC, presenting such a high probability of hypothesis or overfeeding. IC is considered the gold standard to assess energy expenditure, but not always available and also available. Recent guidelines from the American Society of Parenteral and Enteral Nutrition for nutritional support of critically ill children suggest that IC measures may be used when possible in pediatric patients suspected of metabolic disorders or malnutrition, according to criteria list that can lead to metabolic instability, becoming even less widely used standardized predictive equations. Although standard use of $\mathrm{Cl}$ is limited due to equipment availability, staff and cost, a review of commonly used measurements is used. The objective of this study was to compare measures of expenditure and through $\mathrm{Cl}$ with predictive equations. Methods: This prospective observational study was conducted in a pediatric oncology UTI from January to June 2019. Data were collected on gender, age and anthropometry from 18 patients who were within the inclusion test. As energy requirements were calculated by the routine predictive equations, such as Holliday-Segar, ASPEN and Dietary Reference Intake (DRI), IC has already been applied to hemodynamically stable mechanically ventilated patients with FiO, below $60 \%$ and was used without ventilation for 2 hours; after 2 hours the energy expenditure was read on the monitor. The data were analyzed descriptively and by the concordance index between formulas and IC, being analyzed through Spearman test and agreeing through intraclass correlation, being adopted significance 0.05 . Results: Eighteen patients were included, $77 \%$ female, with prevalence of eutrophic nutritional status (44.4\%), while overweight, underweight and malnourished patients, $50.1 \%$. The correlations of the predictive formulas with the IC, Holliday-Segar (0.461-p=0.054), DRI $(0.428-p=0.076)$ and ASPEN $(0.232-p=0.354)$, are therefore the most consistent with the gold standard. Holliday-Segar's formula. Conclusion: The IC should be used, if available, when the patient is within inclusion criteria, but due to the higher price, the predictive formulas that can be used, considering the importance of nutritional needs.

1. Nutricionista Especialista em Nutrição Hospitalar pelo Hospital de Base; Residente em Oncologia Pediátrica pelo Hospital de Câncer de Barretos, Barretos, SP, Brasil.

2. Nutricionista Especialista em Oncologia Pediátrica pelo Hospital de Câncer de Barretos, Barretos, SP, Brasil.

3. Fisioterapeuta Residente em Oncologia Pediátrica pelo Hospital de Câncer de Barretos, Barretos, SP, Brasil.

4. Fisioterapeuta no Hospital do Câncer de Barretos; Mestre e Doutora em Bases Gerais da Cirurgia - UNESP, Barretos, SP, Brasil.

5. Enfermeira Especialista em Terapia Intensiva pela FAMERP; Especialista em Gestão de Enfermagem pela Unifesp; Docente no ensino técnico no SENAC; Mestranda em Oncologia pela Fundação PIO XII, Barretos, SP, Brasil.

6. Médica Intensivista Pediátrica no Hospital de Câncer de Barretos; Especialista em Pediatria e Terapia Intensiva Pediátrica; Mestranda em Oncologia na Fundação Pio XII; Barretos, SP, Brasil.

7. Nutricionista no Hospital de Câncer de Barretos; Mestre em Ciências da Saúde; Barretos, SP, Brasil. 


\section{INTRODUÇÃO}

O metabolismo da criança e do adolescente saudável pode ser influenciado por vários fatores, dentre eles idade, sexo, estado nutricional, composição corporal, hormônios, atividade física e ambiente. No entanto, ainda há controvérsias em relação às alterações metabólicas (hipermetabolismo, anorexia, catabolismo) que um paciente pediátrico com câncer pode desenvolver. Além disso, quando comparados aos adultos são mais vulneráveis à perda de peso e à piora do estado nutricional.

O impacto do câncer no estado nutricional desses pacientes pode ser variável por diferentes aspectos, como tipo de tumor, localização, tipo de tratamento e suas respectivas complicações ${ }^{1}$. As terapias antineoplásicas, como quimioterapia, radioterapia, cirurgia, transplante de células-tronco hematopoiéticas, ou a combinação destas, podem contribuir para essas alterações? ${ }^{1}$.

Os pacientes pediátricos são particularmente mais vulneráveis à desnutrição, quando comparados aos adultos. Estudos demonstram que de $6 \%$ a $50 \%$ dos pacientes oncológicos pediátricos apresentam algum grau de desnutrição no momento do diagnóstico, por isso suas necessidades energéticas são elevadas ${ }^{1,2}$.

A desnutrição pode estar correlacionada com maior número de internações, maior propensão a infecções, menor resposta às terapias, influenciando diretamente nas taxas de sobrevida².

O paciente oncológico grave apresenta uma situação de risco nutricional, por estar, na maioria das vezes, em estado catabólico, levando à depleção de massa muscular e ao inadequado estado nutricional. Esses fatores podem agravar o comprometimento da imunidade, reduzir a tolerância aos tratamentos, piorando a recuperação clínica perante os procedimentos invasivos ${ }^{3}$.

$\mathrm{Na}$ terapia intensiva, a desnutrição permanece prevalente entre crianças admitidas nas Unidades de Terapia Intensiva Pediátrica (UTIP) e a prevalência desta na população pediátrica criticamente enferma varia de $10 \%$ a $45 \% 4,5$, sendo assim um desafio para a equipe multidisciplinar determinar e prover adequada energia para otimizar a síntese tissular e a função imune $e^{1,2,6}$.

A determinação do gasto energético individualizado é de suma importância, pois fornece necessidades precisas durante o tratamento, minimizando o risco nutricional, melhorando a sobrevida dos pacientes ${ }^{7}$. Crianças criticamente enfermas, inadequadamente alimentadas, com subalimentação ou com superalimentação, podem ter aumento em sua morbidade e mortalidade e as crianças adequadamente alimentadas têm uma melhora na estabilidade fisiológica e melhores desfechos clínicos ${ }^{1-3,8}$.
Diferentes são as equações para avaliar o gasto energético do paciente crítico, essas são baseadas no gasto energético em repouso (GER) para crianças saudáveis?. O GER é definido como a necessidade de calorias requeridas pelo corpo em repouso durante um período, representando $70 \%$ a $80 \%$ das calorias usadas pelo corpo, sendo o GER a taxa metabólica basal que define a energia liberada para manter o funcionamento fisiológico basal normal. Crianças gravemente enfermas têm variação no GER e isto pode ser atribuído ao ambiente de UTIP com temperatura controlada, à imobilidade, ao uso de sedativos ou relaxantes musculares, à ventilação mecânica, à própria doença de base, como, por exemplo, queimaduras, doenças cardíacas e sepse, onde o gasto energético é elevado e há uso de drogas vasoativas ${ }^{3,10}$.

As equações preditivas são baseadas em dados de gasto energético de pacientes saudáveis, para melhor definição clínica, portanto, quando aplicadas a pacientes críticos podem apresentar limitações. Além disso, são controversas, e possuem baixa acurácia quando comparadas à calorimetria indireta $(\mathrm{Cl})$, apresentando, assim, grande probabilidade de hipo ou hiperalimentação ${ }^{3}$. As crianças criticamente doentes possuem gravidades imprevisíveis, além de particularidades na resposta metabólica. Devido a isso, podem resultar na falha de estimativa precisa das necessidades nutricionais ${ }^{4}$.

Dessa forma, as equações preditivas não têm boa acurácia para calcular as necessidades energéticas em crianças internadas em UTIP devido às alterações metabólicas destas crianças e o uso destas fórmulas pode ainda estar associado a maior tempo de internação na UTIP, por complicações como a subalimentação e a superalimentação ${ }^{2,9}$. Por isso, as diretrizes da ASPEN (Sociedade Americana de Nutrição Parenteral e Enteral) e ESPEN (Sociedade Europeia de Nutrição Enteral e Parenteral) sugerem que o suporte nutricional da criança gravemente doente deva suprir as necessidades obtidas a partir das medidas de $\mathrm{Cl}^{8}$.

$\mathrm{A} \mathrm{Cl}$ é considerada padrão-ouro para avaliar o gasto energético, mas nem sempre está disponível e também possui limitações ${ }^{11}$. Trata-se de um método que determina as necessidades nutricionais a partir do consumo de oxigênio e da produção de gás carbônico obtidos por meio do ar inspirado e expirado pelos pulmões. Tal método possibilita identificar a metabolização pelo organismo de substratos energéticos e sua origem ${ }^{12}$.

$\mathrm{A} \mathrm{Cl}$ avalia a quantidade de calor gerada indiretamente, de acordo com a quantidade e o padrão de utilização dos substratos e da produção de subprodutos. Esta energia mensurada decorre de energia química que é liberada a partir de nutrientes por meio da oxidação de substratos alimentares. 
Nutrientes à base de carbono são convertidos em dióxido de carbono, água e calor na presença de oxigênio $\left(\mathrm{O}_{2}\right)^{12}$.

Embora o uso padrão da $\mathrm{Cl}$ seja limitado devido à disponibilidade do equipamento e de equipe e ao custo, as medições tornaram-se mais precisas ${ }^{10}$.

Dessa maneira, o presente estudo objetivou comparar e avaliar o índice de concordância das medidas de gasto energético através da $\mathrm{Cl}$ com as equações preditivas de pacientes internados em uma UTIP de pacientes oncológicos.

\section{MÉTODO}

Trata-se de um estudo prospectivo, de caráter observacional, realizado em uma UTIP oncológica. Foram incluídos pacientes no período de janeiro a junho de 2019.

Foram coletados dados como sexo, idade e antropometria relatada ou estimada, peso, estatura, circunferência do braço, dobra cutânea tricipital e área muscular do braço, e avaliadas suas adequações.

Foi avaliada a adequação do estado nutricional dos pacientes e, a partir da antropometria (peso e estatura), foi calculado o índice de massa corporal (IMC), o IMC/idade e, em seguida, associado às curvas da $\mathrm{OMS}^{5}$, sendo estes determinados através de percentil (Tabela 1).

As necessidades energéticas de cada paciente foram calculadas pelas equações preditivas utilizadas na rotina do serviço, dentre elas Holliday-Segar (Tabela 2), ASPEN (Tabela 3) e Dietary Reference Intake (DRI) (Tabela 4).

Um dos métodos utilizados na prática clínica para 0 estabelecimento das necessidades nutricionais de energia é de Holliday-Segar, publicado em 1957, obtido através de um estudo realizado em crianças hospitalizadas. Para o seu cálculo, é utilizado apenas o peso do paciente.

\begin{tabular}{lc}
\hline Tabela 1 - Classificação pelas curvas da OMS, nos índices IMC/I. \\
\hline IMC/I & \\
\hline Acima do p97 & Obesidade \\
Entre p97 e p85 & Sobrepeso \\
Entre p85 e p15 & Eutrofia \\
Entre p15 e p3 & Baixo peso \\
Abaixo do p3 & Desnutrição \\
\hline
\end{tabular}

Fonte: $\mathrm{OMS}^{5} . \mathrm{IMC} / \mathrm{I}=$ índice de massa corporal por idade.

\begin{tabular}{|c|c|}
\hline Holliday-Segar - 1957 & \\
\hline Crianças de $0 \mathrm{~kg}$ a $10 \mathrm{~kg}$ & $100 \mathrm{kcal} / \mathrm{kg}$ \\
\hline Crianças de $10 \mathrm{~kg}$ a $20 \mathrm{~kg}$ & $\begin{array}{c}1.000 \mathrm{kcal}+50 \mathrm{kcal} / \mathrm{kg} \text { para cada } \mathrm{kg} \\
\text { acima de } 10 \mathrm{~kg}\end{array}$ \\
\hline Crianças com mais de $20 \mathrm{~kg}$ & $\begin{array}{c}1.500 \mathrm{kcal}+20 \mathrm{kcal} / \mathrm{kg} \text { para cada } \mathrm{kg} \\
\text { acima de } 20 \mathrm{~kg}\end{array}$ \\
\hline
\end{tabular}

\begin{tabular}{|c|c|}
\hline \multicolumn{2}{|l|}{ ASPEN - 2002} \\
\hline Idade (anos) & $\mathrm{kg} /$ peso \\
\hline De 0 a 1 & 90 a 120 \\
\hline De 1 a 7 & 75 a 90 \\
\hline De 7 a 12 & 60 a 75 \\
\hline De 12 a 18 & 30 a 60 \\
\hline De 18 a 25 & 25 a 30 \\
\hline
\end{tabular}

Tabela 4 - Fórmula de necessidade energética por DRI.

\begin{tabular}{lc}
\hline Dietary Reference Intake (DRI) - 2006 \\
\hline De 0 a 3 meses & $(89$ x peso $(\mathrm{kg})-100)+175$ \\
De 4 a 6 meses & $(89$ x peso $(\mathrm{kg})-100)+56$ \\
De 7 a 12 meses & $(89 \times$ peso $(\mathrm{kg})-100)+22$ \\
De 13 a 35 meses & $(89 \times$ peso $(\mathrm{kg})-100)+20$ \\
\hline
\end{tabular}

Tabela 5 - Prevalência de sexo de pacientes incluídos.

\begin{tabular}{ccc}
\hline Sexo & Número de pacientes & Porcentagem \\
\hline Feminino & 14 & $77,8 \%$ \\
Masculino & 4 & $22,2 \%$ \\
\hline
\end{tabular}

Tabela 6 - Diagnóstico nutricional dos pacientes incluídos.

\begin{tabular}{lcc}
\hline Variável & Número de pacientes & Porcentagem \\
\hline Desnutrição & 3 & $16,7 \%$ \\
Baixo Peso & 3 & $16,7 \%$ \\
Eutrofia & 8 & $44,4 \%$ \\
Sobrepeso & 3 & $16,7 \%$ \\
Obesidade & 1 & $5,6 \%$ \\
\hline
\end{tabular}

Tabela 7 - Valores das necessidades energéticas pelas equações preditivas e Cl em quilocaloria.

\begin{tabular}{lcccc}
\hline & Média & Mediana & Mínimo & Máximo \\
\hline VET Holliday & 1728,9 & 1745,0 & 900 & 2385 \\
VET ASPEN & 1753,7 & 1796,0 & 855 & 2925 \\
VET DRI & 1556,3 & 1648,0 & 765 & 2198 \\
VET Calorimetria & 1506,2 & 1257,5 & 767 & 3387 \\
\hline VET= Valor Energético Total. & & & &
\end{tabular}

Tabela 8 - Índice de correlação de fórmulas preditivas com a Cl.

\begin{tabular}{lcc}
\hline Fórmula Preditiva & IC & p Valor \\
\hline Holliday-Segar & 0,461 & 0,054 \\
DRI & 0,428 & 0,076 \\
ASPEN & 0,232 & 0,354 \\
\hline DRI= Dietary Reference Intakes; ASPEN= Sociedade Americana de Nutrição Parenteral e \\
Enteral.
\end{tabular}


As necessidades de energia da criança e do adolescente também podem ser obtidas através da DRI, que são valores de referência por faixa etária para estimar a ingestão de nutrientes. A DRI foi estabelecida para os habitantes dos Estados Unidos e Canadá, e foi desenvolvida para população sadia.

$E$, por fim, a calorimetria, método esse considerado padrão-ouro, consegue avaliar por meio da ventilação mecânica, em pacientes estáveis hemodinamicamente, com $\mathrm{FiO}_{2}$ menor de 60\%, a necessidade energética.

Critérios de inclusão: foram incluídos no estudo pacientes de 2 a 18 anos completos, com diagnóstico de câncer pediátrico, que necessitaram de terapia intensiva e ventilação mecânica no período estabelecido do estudo, estáveis hemodinamicamente, com $\mathrm{FiO}_{2}$ menor de $60 \%$, sem outro comprometimento pulmonar.

O módulo da calorimetria foi acoplado ao ventilador do paciente por 2 horas; após 2 horas, foi feita a leitura do gasto energético no monitor, os valores foram anotados e a calorímetro retirado.

Os dados foram coletados na plataforma Research Electronic Data Capture (REDCAP), diariamente.

\section{Análise estatística}

Os dados foram analisados pelo índice de concordância entre as equações preditivas e a $\mathrm{Cl}$, sendo analisada por meio do teste de Spearman e a concordância, por meio da correlação intraclasse, foi adotado significância 0,05. Outros dados foram analisados, realizadas com auxílio dos programas Excel e Statistical Package for the Social Sciences (SSPS) versão 21.0, sendo tratados através da estatística descritiva básica (percentuais).

\section{RESULTADOS}

Foram incluídos 18 pacientes que atenderam aos critérios de inclusão. Apresentaram média de idade de 10 anos. A prevalência do sexo foi o feminino (Tabela 5).

O estado nutricional prevalente foi eutrofia, seguido de desnutrição, baixo peso e sobrepeso, os quais somam $50,1 \%$ (Tabela 6). Foram coletados, também, outros dados antropométricos, porém não relacionados à necessidade energética.

Os valores do gasto energético, calculados por meio de equações preditivas e $\mathrm{Cl}$, tiveram valores médios de 1506,2 kcal a 1728,9 kcal. Dentre eles, o valor energético mínimo foi de 900 kcal, utilizando Holliday-Segar, e máximo 3387 kcal, utilizando $\mathrm{Cl}$ (Tabela 7).

A Tabela 8 apresenta o índice de correlação das fórmulas preditivas com a Cl: foram Holliday-Segar $(0,461$ - $p=0,054)$, DRI $(0,428-p=0,076)$ e ASPEN $(0,232-p=0,354)$.
Considerando o índice de concordância (IC) que quanto mais próximo de 1, maior é a correlação entre a equação e a calorimetria, considera-se que a fórmula preditiva com maior concordância ao padrão ouro pelo $p$ valor mais próximo de 0,05 e correlação pouco maior que a DRI foi a fórmula de Holliday-Segar, com o IC =0,461 e $p=0,054$.

\section{DISCUSSÃO}

Grande parte dos estudos com calorimetria inclui em suas amostras de pacientes internados, em sua maioria, indivíduos do sexo masculino. Entretanto, no presente estudo, a maior parte dos pacientes era do sexo feminino.

Assim como Caldeira et al. ${ }^{9}$ e Barreto et al. ${ }^{13}$, que descreveram o perfil nutricional de pacientes oncológicos internados, onde a taxa de eutrofia era prevalente, no presente estudo, $44 \%$ dos pacientes analisados apresentavam-se eutróficos.

Outro estudo concluiu que a $\mathrm{Cl}$ também possui limitações, além de demandar cuidados na interpretação dos dados. Durante o desenvolvimento do estudo, foi visto que alguns pacientes não se beneficiaram da calorimetria, e houve a necessidade de sua retirada, pois após a instalação da mesma os pacientes apresentaram retenção de $\mathrm{CO}_{2}$. Por isso, sugere-se que, na ausência de calorimetria, sejam utilizadas as equações preditivas para estimar as necessidades energéticas, mas que demandam cuidado no momento da interpretação. Sendo assim, alguns centros ainda utilizam as equações estimadas para obtenção das necessidades de energia das crianças ${ }^{14}$.

Além das fórmulas preditivas analisadas, um estudo realizado na Europa, em 111 unidades de UTIP, cujo objetivo era conhecer os métodos empregados para estimar as necessidades nutricionais de energia, encontrou que peso e idade eram utilizados pela maioria dos profissionais. Além deste método, demonstraram que as equações preditivas eram utilizadas por cerca de 30\% dos profissionais, seguido de uma minoria que utilizava um monitor metabólico de forma intermitente para avaliação do gasto energético ${ }^{15}$.

Suman et al. ${ }^{16}$, que avaliaram 91 crianças gravemente queimadas, hipermetabólicas, também compararam o GER pela $\mathrm{Cl}$ e as fórmulas preditivas. Utilizaram as equações de Schonfield, peso e estatura, Harris-Bennedict, equação da Organização de Comida e Agricultura, Organização Mundial de Saúde e Universidade das Nações Unidas. Esses autores verificaram que o GER predito pelas equações foi inferior ao encontrado na $\mathrm{Cl}$, a qual apresenta melhor acurácia. $\bigcirc$ mesmo é descrito por Sion-Sarid et al. ${ }^{17}$, em outro artigo de revisão de terapia nutricional em crianças internadas em UTIP, que recomendam a avaliação do GER pelo uso da $\mathrm{Cl}$. 
Sendo assim, os trabalhos levantados vão de encontro com as recomendações das ESPEN e ASPEN que sugerem que, quando possível, deve ser realizada a calorimetria, considerada padrão-ouro, para avaliação das necessidades energéticas. Quando esse método não estiver disponível, faz-se necessário o uso das fórmulas preditivas, sendo assim, este trabalho sugere que dentre as equações a que mais se correlaciona com a $\mathrm{Cl}$ é a Holliday-Segar.

\section{CONCLUSÃO}

Dessa forma, a $\mathrm{Cl}$ deve ser utilizada quando disponível no serviço e quando o paciente estiver dentro dos critérios de inclusão, porém pelo seu alto custo e suas limitações, as fórmulas preditivas podem ser utilizadas, considerando a facilidade de aplicação e a importância das necessidades nutricionais individualizadas para o tratamento e sobrevida do paciente.

Entretanto, devido às limitações do tamanho da amostra do atual trabalho e à escassez de técnicas para avaliação da composição corporal em pediatria, estudos com maiores tamanhos amostrais e que utilizem o padrão ouro para avaliar a composição corporal são necessários, a fim de elucidar qual equação se assemelha mais ao padrão-ouro.

\section{REFERÊNCIAS}

1. Viani K, Oliveira V, Nabarrete J, Silva APA, Feferbaum R. Nutrição e câncer infantojuvenil. Manole: Barueri; 2017.

2. Garófolo A. Diretrizes para terapia nutricional em crianças com câncer em situação crítica. Rev Nutr. 2005;18(4):513-27.

3. Toledo DO, Piovacari SMF, Horie LM, Matos LBN, Castro MG, Ceniccola, GD, et al. Campanha "Diga não à desnutrição": 11 passos importantes para combater a desnutrição hospitalar. BRASPEN J. 2018;33(1):86-100.

4. Taylor RM, Cheeseman P, Preedy V, Baker AJ, Grimble G. Can energy expenditure be predicted in critically ill children? Pediatr Crit Care Med. 2003;4(2):176-80.

5. WHO Multicentre Growth Reference Study Group. WHO child growth standards: methods and development. Length/heightfor-age, weight-for-age, weight-for-length, weight-for-height and body mass index-for-age. Geneva: World Health Organization; 2006.

6. Jotterand Chaparro C, Moullet C, Taffé P, Laure Depeyre J, Perez MH, Longchamp D, et al. Estimation of resting energy expenditure using predictive equations in critically ill children: results of a systematic review. JPEN J Parenter Enteral Nutr. 2018;42(6):976-86.

7. Schwegler I, von Holzen A, Gutzwiller JP, Schlumpf R, Mühlebach S, Stanga Z. Nutritional risk is a clinical predictor of postoperative mortality and morbidity in surgery for colorectal cancer. Br J Surg. 2010;97(1):92-7.

8. Mehta NM, Skillman HE, Irving SY, Coss-Bu JA, Vermilyea S, Farrington EA, et al. Guidelines for the provision and assessment of nutrition support therapy in the pediatric critically ill patient: Society of Critical Care Medicine and American Society for Parenteral and Enteral Nutrition. JPEN J Parenter Enteral Nutr. 2017;41(5):706-42.

9. Caldeira PT, Ávila e Silva L, Rocha AMS, Amaral DA, Gregório EL. Avaliação do perfil nutricional dos pacientes internados na oncologia pediátrica do Hospital Santa Casa de Misericórdia de Belo Horizonte-MG. HU Revista. 2016;42(1):75-81.

10. Huhmann MB, August DA. Review of American Society for Parenteral and Enteral Nutrition (ASPEN) clinical guidelines for nutrition support in cancer patients: nutrition screening and assessment. Nutr Clin Pract. 2008;23(2):182-8.

11. Wichansawakun S, Meddings L, Alberda C, Robbins S, Gramlich L. Energy requirements and the use of predictive equations versus indirect calorimetry in critically ill patients. Appl Physiol Nutr Metab. 2015;40(2):207-10.

12. Diener JRC. Calorimetria indireta. Rev Assoc Med Bras. 1997;43(3): 245-53.

13. Barreto ABR, Haack A, Santos ACS, Silva APR. Perfil nutricional de pacientes pediátricos portadores de câncer, internados no Hospital da Criança de Brasília. Com Ciências Saúde. 2013;24(4):315-20.

14. Walker RN, Heuberger RA. Predictive equations for energy needs for the critically ill. Respir Care. 2009;54(4):509-21.

15. Van der Kuip M, Oosterveld MJ, van Bokhorst-de van der Schueren MAE, de Meer K, Lafeber HN, Gemke RJBJ. Nutritional support in 111 pediatric intensive care units: a European survey. Intensive Care Med. 2004;30(9):1807-13.

16. Suman OE, Spies RJ, Celis MM, Mlcak RP, Herndon DN. Effects of a 12-wk resistance exercise program on skeletal muscle strength in children with burn injuries. J Appl Physiol (1985). 2001;91(3):1168-75.

17. Sion-Sarid R, Cohen J, Houri Z, Singer P. Indirect calorimetry: a guide for optimizing nutritional support in the critically ill child. Nutrition. 2013;29(9):1094-9.

Local de realização do estudo: Hospital de Câncer de Barretos, Barretos, SP, Brasil.

Conflito de interesse: As autoras declaram não haver.

Foi apresentado como trabalho de destaque no XXIII Congresso Brasileiro de Nutrição Parenteral e Enteral, entre 20 a 23 de outubro de 2019, em Foz do Iguaçu-PR. 